results for operators not assumed positive by means of a reduction procedure [4] and the present theorems.

We are indebted to the work of Eberhard Hopf for suggesting that a resolution of this type is possible.

\title{
BibLIOGRAPHY
}

1. R. V. Chacon and D. S. Ornstein, $A$ general ergodic theorem, Illinois J. Math. 4 (1960), 153-160.

2. R. V. Chacon, The influence of the dissipative part of a general Markov process, Proc. Amer. Math. Soc. 11 (1960), 957-961.

3. - Identification of the limit of operator averages, J. Math. Mech. (to appear).

4. - Convergence of operator averages, (to appear).

BROWN UNIVERSITY

\section{TCHEBYCHEFF QUADRATURE IS POSSIBLE ON THE INFINITE INTERVAL ${ }^{1}$}

\author{
BY J. L. ULLMAN
}

Communicated by J. L. Doob, May 29, 1962

The purpose of this announcement is to state a theorem on Tchebycheff quadrature which answers a question posed in [1], and to discuss the proof. Complete details will appear elsewhere.

\section{Tchebycheff quadrature.}

Definition 1.1. A unit mass distribution on $(-\infty, \infty)$ possessing moments of all positive integer order will be said to belong to class $D$.

Definition 1.2. Let $\psi$ be an element of $D$ and $n$ a positive integer. We refer to the equations

$$
\frac{1}{n} \sum_{i=1}^{n} x_{i, n}^{k}=\int x^{k} d \psi, \quad k=1, \cdots, n
$$

as the equations $(\psi, n)$. These equations admit a solution $x_{1, n}, \cdots$, $x_{n, n}$ which is unique up to permutation of the first index.

Definition 1.3. $T$ quadrature is said to be possible for an element $\psi$ of $D$ if equations $(\psi, n)$ have real solutions for every positive integer $n$. If $T$ quadrature is possible for $\psi$ it is called a $T$ distribution.

1 This research was supported in part by National Science Foundation Grant No. G19654. 
Lemma 1.1 [1]. The mass set of a T distribution lies on a finite interval.

Definition 1.4. $T_{1}$ quadrature is said to be possible for an element $\psi$ of $D$ if the equations $(\psi, n)$ do not have real solutions for every positive integer $n$, but do have real solutions for an infinite number of positive integers. If $T_{1}$ quadrature is possible for $\psi$ it is called a $T_{1}$ distribution. The values of $n$ for which equations $(\psi, n)$ have real solutions are called the $T$ set of $\psi$. If either $T$ or $T_{1}$ quadrature is possible for $\psi$ in $D$, we say Tchebycheff quadrature is possible.

We are now led to the question raised in [1], namely, is there a $T_{1}$ distribution whose mass does not lie on a finite interval, or in other words, is Tchebycheff quadrature possible on the infinite interval? Evidence is produced there that this is not so, since it is shown that if a $T_{1}$ distribution exists whose mass does not lie on a finite interval, its $T$ set would have very large gaps.

THeOREM 1.1. There is a $T_{1}$ distribution whose mass does not lie on a finite interval.

2. Discussion of proof. Lemmas are stated here but not proved. Comments are added which will indicate how the theorem is proved.

Definition 2.1. A simple distribution of degree $n$ is a unit mass distribution consisting of equal masses at $n$ distinct points.

Definition 2.2. Let $\psi, \psi^{\prime}$ be two elements of $D$, and let $m_{k}, m_{k}^{\prime}$ denote the moments $\int x^{k} d \psi, \int x^{k} d \psi^{\prime}$, respectively, $k=1, \cdots$. Let $n$ be a positive integer. Then

$$
\left\|\psi-\psi^{\prime}\right\|_{n}
$$

is defined as

$$
\max \left\{\left|m_{1}-m_{1}^{\prime}\right|, \cdots,\left|m_{n}-m_{n}^{\prime}\right|\right\} .
$$

Lemma 2.1. Let $\psi$ be a simple distribution of degree $n$. There is a number $\epsilon>0$, called a proximity number of $\psi$, such that if

$$
\left\|\psi-\psi^{\prime}\right\|_{n} \leqq \epsilon,
$$

where $\psi^{\prime}$ is any element of $D$, then the equations $\left(\psi^{\prime}, n\right)$ have $n$ distinct real solutions.

Lemma 2.2. There is an element $\psi$ of $D$ whose mass is not contained in a finite interval, and an infinite sequence of simple distributions $\psi_{k}$ of degree $n_{k}$ and with proximity numbers $\epsilon_{k}, k=1, \cdots$, where the $n_{k}$ tend to infinity, such that

$$
\left\|\psi-\psi_{k}\right\|_{n_{k}} \leqq \epsilon_{k}, \quad k=1, \cdots
$$


Comment 1. The condition (2.1) implies that equations $\left(\psi, n_{k}\right)$ have real solutions for $k=1, \cdots$, so that $\psi$ is a $T_{1}$ distribution.

Lemma 2.3. Let $\left\{0_{i}\right\}, i=1, \cdots$, be a family of nonoverlapping, finite intervals on the real axis whose union does not lie in a finite inter$v a l$. There is a sequence of simple distributions $\psi_{k}$ of degree $n_{k}$ and proximity numbers $\epsilon_{k}, k=1, \cdots$, where $n_{k}$ tends to infinity, such that

$$
\int_{0_{k}} d \psi_{k+p} \geqq \gamma_{k}>0, \quad k=1, \cdots, p=0, \cdots,
$$

and

$$
\left\|\psi_{k+p}-\psi_{k}\right\|_{n_{k}} \leqq \epsilon_{k}, \quad k=1, \cdots, p=1, \cdots .
$$

Comment 2. From the $\psi_{k}$ we can extract a sequence whose limit $\psi$ is in $D$. This distribution $\psi$ and the $\psi_{k}$ of this lemma satisfy the conditions of Lemma 2.2. The mass of $\psi$ is not on a finite interval because of (2.2), and (2.3) leads to (2.1).

Comment 3. In constructing the sequence $\psi_{k}$ we proceed in a stepwise fashion, constructing $\psi_{k+1}$ from $\psi_{k}$ in two stages. $\psi_{k}$ has all its mass on the sets $0_{1}, \cdots, 0_{k}$. We move some mass from $0_{k}$ to $0_{k+1}$, thus creating a mass distribution $\psi_{k}^{\prime}$. We then split each mass of $\psi_{k}^{\prime}$ into a number of equal masses, locating them close to the mass in which they originated. This can be done so that $\psi_{k+1}$ is simple and has all its mass on $0_{1}, \cdots, 0_{k+1}$.

\section{REFERENCES}

1. H. S. Wilf, The possibility of Tchebycheff quadrature on infinite intervals, Proc. Nat. Acad. Sci. U.S.A. 47 (1961), 209-213.

University of MichigaN 